Gynäkologe 2015 · 48:198-199

DOI 10.1007/s00129-014-3394-0

Online publiziert: 30. Januar 2015

(c) Springer-Verlag Berlin Heidelberg 2015
N. Maass ${ }^{1} \cdot$ W. Janni ${ }^{2}$

${ }^{1}$ Frauenklinik für Gynäkologie und Geburtsmedizin, Perinatalzentrum Level I, Universitätsklinikum Aachen

${ }^{2}$ Frauenklinik, Universitätsklinikum Ulm

\title{
Multimodale Behandlungskonzepte der Endometriose
}

Liebe Kolleginnen und Kollegen, in dieser Ausgabe von Der Gynäkologe stehen multimodale Behandlungskonzepte der Endometriose im Fokus. Die verschiedenen Artikel sollen Einblicke bieten in

- rezente Erkenntnisse zu Diagnostik, Pathogenese, operativen und konservativen Therapiekonzepten,

- zu aktuellen Fragen der Klassifikation der Endometriose,

- zur besonderen Situation bei Kinderwunsch und Endometriose sowie

- zur Frage nach erhöhtem Risiko für assoziierte maligne Erkrankungen.

Endometriose ist eine der häufigsten gutartigen Erkrankung in unserem Fachgebiet und stellt durch die klinisch vielfältige, oft sehr unterschiedliche Symptomatik schon in der Diagnostik eine große Herausforderung dar. Trotz umfangreicher Bemühungen - Etablierung spezialisierter Endometriosezentren, Öffentlichkeitsarbeit, Aufklärung und Information von medizinischem Fachpersonal und Ärzten verschiedener Fachgebiete - verzögern sich die Diagnose und damit die Möglichkeit der adäquaten Behandlung dieser Erkrankung weiterhin um durchschnittlich etwa 7 Jahre nach Auftreten erster Beschwerden. Hier besteht weiterhin Handlungsbedarf.

Neben der Betreuung vieler Patientinnen in spezialisierten Endometriosezentren haben die stetige Weiterentwicklung der endoskopischen Operationsverfahren und die schwerpunktmäßige Ausbildung der Operateure sicherlich zur verbesserten Diagnostik und optimierten operativen Therapie beigetragen. Die große Kunst in der erfolgreichen Behandlung der Endometriose bleibt es weiterhin, eine Balance zwischen ,watchful waiting", konsequenter Analgesie, hormoneller Therapie, operativer Intervention und die Kombination aus Operation und medikamentöser Therapie zu finden. Eine kurative Therapie existiert bisher nicht, die etablierten Therapieverfahren verfolgen vorwiegend einen symptomatischen Ansatz.

\section{I) Nach wie vor wird eine Endometriose oft erst spät diagnostiziert}

Neben der operativen Sanierung ist die medikamentöse Behandlung eine wichtige Therapiesäule. Ihr vorrangiges Ziel ist das Erreichen eines hypoöstrogenen Zustands durch verschiedene hormonelle Therapeutika, insbesondere orale Kontrazeptiva, Gestagene und GnRH-Analoga. Heute stehen zusätzlich experimentelle Verfahren zur Verfügung, wie Aromataseinhibitoren, GnRH-Antagonisten, selektive Östrogenrezeptor- (SERM) und selektive Progesteronrezeptormodulatoren (SPRM), Angiogeneseinhibitoren sowie Immunmodulatoren. Alternative Ansätze, wie die multimodale Schmerztherapie, und komplementäre Verfahren, wie z.B. Akupunktur, Physiotherapie und Phytotherapie, ergänzen die Behandlungsmöglichkeiten.

D) Kein Klassifikationssystem
bildet alle Aspekte
der Endometriose
multidimensional ab

Um die Erkrankung Endometriose zu beschreiben und vergleichbar zu machen, gab es diverse Versuche, möglichst viele Aspekte dieser Erkrankung in die Klassifikation einfließen zu lassen. Die derzeit gebräuchlichste Klassifikation ist die 1997 revidierte Form der American Society of Reproductive Medicine (rASRM). Da diese rASRM-Klassifikation die Prognose der Erkrankung für Sterilitätspatienten hinsichtlich ihres Kinderwunsches nicht abbildet, wurde der Endometriosis Fertility Index (EFI) entwickelt. Er schließt zusätzlich sowohl anatomische als auch anamnestische Parameter der jeweiligen Patientin ein und findet zunehmend Anwendung. In beiden Klassifikationssystemen ist jedoch die tief infiltrierende Endometriose nicht berücksichtigt. Hierfür wurde die ENZIAN-Klassifikation entwickelt, die in Anlehnung an das TNM-System der malignen Tumoren den Schweregrad der Endometriose darstellt. Bis heute gibt es kein Klassifikationssystem, das alle Aspekte der Endometriose multidimensional abbilden kann, und es bleibt weiterhin schwierig, einzelne Fälle zu vergleichen und das klinische Management abzustimmen.

Die Rezidivrate für eine therapierte Endometriose liegt zwischen 5 und $60 \%$ 
und ist wesentlich abhängig vom ganzheitlichen Management sowie von der operativen Qualität. Dies unterstreicht einmal mehr, wie wichtig klare Konzepte und multimodale Behandlungsstrategien für das erfolgreiche Management dieser häufig chronisch rezidivierenden Erkrankung sind.

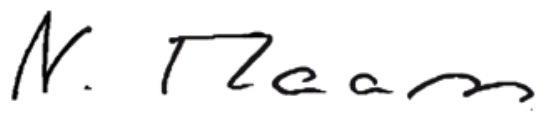

Prof. Dr. Nicolai Maass

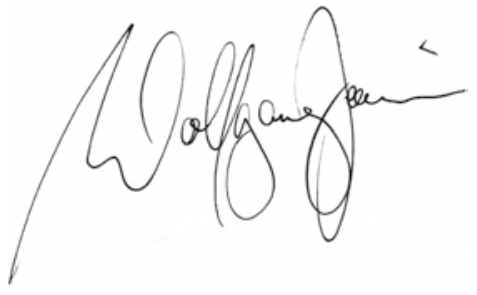

Prof. Dr. Wolfgang Janni

\section{Korrespondenzadresse}

\section{Prof. Dr. N. Maass}

Frauenklinik für Gynäkologie und

Geburtsmedizin, Perinatalzentrum Level I,

Universitätsklinikum Aachen

Pauwelsstr. 30, 52074 Aachen

nmaass@ukaachen.de

Interessenkonflikt. N. Maass und W. Janni geben an, dass kein Interessenkonflikt besteht.

\section{Petru, E., Jonat, W., Fink, D., Köchli, O.R. \\ (Hrsg.) \\ Praxisbuch Gynäkologische Onkologie}

Berlin Heidelberg New York: Springer 2014, 4. Auflage, 334 S., 48 Abb., (ISBN ISBN 978-3-662-434), 74.00 EUR

Das „Praxisbuch Gynäkologische Onkologie" erscheint jetzt in der 4. Auflage. Alleine diese Tatsache belegt, dass das Konzept der praxisnahen Darstellung der klinischen Aspekte der gynäkologischen Onkologie greift. Der relativ kurze Abstand zwischen den Auflagen (1. Auflage 2005, 3. Auflage 2011) gewährleistet eine hohe Aktualität, die dem raschen Fortschritt auf diesen Gebiet gerecht wird. Besonderen Charme gewinnt das Werk durch die Kooperation von namhaften Autoren aus Österreich, Deutschland und der Schweiz. Wie auch bei der Entwicklung der S3-Leitlinien, die in diesen Buch voll berücksichtig sind, arbeiten die gynäkologischen Onkologen dieser Länder eng zusammen und tragen Sorge, dass diese Disziplin mit einer Zunge spricht.

Die Themen Mammakarzinom, Tumoren des Uterus, des Ovars, der Tuben, des Peritoneums, der Vulva und Vagina, sowie Trophoblasttumoren werden umfassend, aber präzise und praxisnah auf aktuellen Stand beschrieben. Für Leser, denen diese detaillierte Darstellung nicht ausreichend ist, finden sich umfangreiche Literaturangaben. Dem Anspruch der Praxisnähe gerecht werdend, sind gezielt die relevanten medikamentösen Regimes sowie deren Nebenwirkungen und ihre Beherrschung beschrieben. Die Radiotherapie in der gynäkologischen Onkologie wird skizziert. Das Lymphödem, die mögliche Prophylaxe und seine Therapie werden beschrieben. Neben allgemeinen supportiven Maßnahmen werden die Schmerztherapie, die Psychoonkologie, die Rehabilitation, die Störungen der Sexualität unter onkologischer Therapie sowie Ernährungsberatung und Therapie während einer Chemo-/Hormontherapie dargestellt. Besonders beachtenswert ist die Abhandlung der komplementärmedizinischen onkologischen Maßnahmen und die Bewertung ihrer wissenschaftlichen Evidenz. Abgerundet wird das Buch durch einen sehr gelungenen Anhang von „Kochrezepten", in denen die praktische Zubereitung und Anwendung der in der gynäkologischen Onkologie verwendeten Medikamente und die gebräuchlichsten The- rapieschemata für den täglichen Gebrauch dargestellt sind.

Ein rundum gelungenes Werk, das es dem Anfänger ermöglicht, rasch eine hohe und umfassende Kompetenz in der gynäkologischen Onkologie zu erwerben und das auch der "Erfahrene“ zur Aktualisierung seines Wissenstandes oder zum Nachlesen von Details gerne nutzen wird.

G. Emons (Göttingen) 